\title{
Lung Ultrasound to detect cardiopulmonary interactions in acutely ill children
}

\author{
Danilo Buonsenso ${ }^{1}$, Cristina De Rose ${ }^{2}$, Valentina Ferro ${ }^{3}$, Rosa Morello ${ }^{4}$, Anna Maria \\ Musolino $^{1}$, and Piero Valentini ${ }^{5}$ \\ ${ }^{1}$ Ospedale Pediatrico Bambino Gesu \\ ${ }^{2}$ Universita Cattolica del Sacro Cuore Sede di Roma \\ ${ }^{3}$ Bambino Gesu Pediatric Hospital \\ ${ }^{4}$ Fondazione Policlinico Universitario A. Gemelli IRCCS, Rome, Italy. \\ ${ }^{5}$ Department of Pediatrics, Fondazione Policlinico A. Gemelli, Universita' Cattolica del \\ Sacro Cuore, Rome, Italy
}

June 29, 2021

\begin{abstract}
Objective and design: Our prospective observational study is the first study that evaluates the LUS findings of cardiopulmonary interactions in acutely ill children with elevated pro-BNP levels, with the aim of establishing the specific LUS pattern in this category of patients without primary lung diseases.Methodology:We prospectively analyzed epidemiological, clinical, laboratory, instrumental and lung ultrasound parameters in acutely ill children aged 1 month to 18 years admitted to the Department of Pediatrics between March 2020 to August 2020.Among the acutely ill patients evaluated, only patients with pro-BNP $>300$ pg / $\mathrm{ml}$ and who underwent LUS before the start of any treatment were included. They were stratified into three sub-categories based on the diagnosis A) cardiac disease, B) systemic inflammatory disease / sepsis without functional and / or organic alterations of the myocardium and C) systemic inflammatory disease / sepsis and cardiac disease, and were classified into two groups based on the level of pro-BNP.We also enrolled patients belonging to two other categories (patients with primary infectious lung disease and completely healthy patients) analyzing their epidemiological, clinical, laboratory, instrumental parameters and lung ultrasound findings and comparing them with those of acutely ill children.Results and Conclusion: We found that LUS findings in these acutely ill children are different from the ultrasound pattern of other categories of children and in particular 1) children with acute lower respiratory tract infections and 2) healthy infants. The finding in a child of a sonographic interstitial syndrome with multiple, bright, long, separate and non-confluent B-lines / long vertical artefacts deriving from a normal and regular pleural line, in the absence of subpleural consolidations, is strongly predictive of cardiogenic pulmonary edema or pulmonary congestion in the course of systemic inflammatory disease / sepsis.
\end{abstract}

\section{INTRODUCTION AND STUDY AIM}

In the last years, Lung Ultrasound (LUS) has gained a primary role in the diagnosis and management of pleuropulmonary disorders, also in pediatric practice. ${ }^{1-3}$

LUS is a non-invasive, non-ionizing radiation tool and a rapid, affordable, point-of-care imaging modality that allow both real-time diagnosis and follow-up of respiratory diseases. ${ }^{1-3}$ LUS results are immediately available to the clinician, especially in emergency conditions who can therefore immediately orient himself towards a diagnosis so that a quick therapeutic decision can be made.

Several studies performed in the pediatric population have shown that LUS may be better than chest radiography in the diagnosis of community-acquired pneumonia ${ }^{1-3}$, can define the etiology of pneumonia ${ }^{3,4}$ 
and many studies have described and validated LUS pattern or score (based mainly on vertical artifacts and subpleural consolidations) in neonatal respiratory disorders ${ }^{5}$ and bronchiolitis ${ }^{6}$ but also the normal LUS pattern in healthy infants. ${ }^{2}$

The latter and other categories of patients are united by having in common the presence of sonographic interstitial syndrome (SIS): the presence of multiple or focal, patched or diffuse vertical artifacts (B-lines) fanning out from the lung wall interface. ${ }^{7}$ It is in fact a lung ultrasound picture common to various respiratory diseases. ${ }^{2,6,8}$

However, to define the nature and possible origin of SIS, not having many pediatric studies on interpretation of LUS vertical artifacts available, especially as regards the differentiation between cardiogenic pulmonary edema or no inflammatory pulmonary congestion and pulmonary edema of inflammatory origin or fibrosis, up to now it was necessary to refer to the studies performed on adults, having always in mind the knowledge of the patient's clinical background. ${ }^{7-10}$

In particular, according to studies performed on physical models and in adult patients ${ }^{7-10}$, the B lines or vertical artifacts, in the absence of an analysis of their appearance, cannot easily differentiate the cause. Soldati et al. ${ }^{7,8,10}$ have shown that the B lines are heterogeneous entities in terms of aggregation and visual structure, the nature of which is linked to the superficial histological characteristics of their wavelength. Vertical artifacts generated by a fibrotic or inflammatory lung have a different appearance from those generated by cardiogenic edema.

Furthermore, several studies performed on the adult population ${ }^{11-13}$ have shown that LUS now plays a leading role in the work-up of adult patients with suspected cardiogenic pulmonary edema. In fact, the approach that has the best performance is the one that combines chest X-ray and the determination of the pro-BNP level with the early use of LUS. ${ }^{13}$ Recent studies and systemic reviews ${ }^{11,12}$ have shown that LUS is highly sensitive and specific (with a greater sensitivity and specificity than chest x-ray) in early identification of cardiogenic pulmonary edema and in its differentiation from primary pulmonary pathologies in dyspneic geriatric patients (through the evaluation of the B lines). ${ }^{7-13}$

In addition, other studies have shown that the level of pro-BNP is not only related to pulmonary edema from heart failure. ${ }^{15-17}$ In fact, the increase in the value of NT-pro-BNP, with consequent different degree of pulmonary congestion, may also be present in the course of other diseases such as the systemic inflammatory syndrome and / or sepsis also in the absence of organic or functional damage to the myocardium. ${ }^{16,17}$

In the pediatric population, on the other hand, in order to interpret the nature of the SIS picture caused by cardio-pulmonary interactions and not by primary pulmonary pathologies, up to now we have referred to the aforementioned preclinical and clinical studies performed on the adult population. ${ }^{7-13}$ In particular, in the pediatric population a study has never yet been performed which aims to define the lung ultrasound pattern in acutely ill children (with heart disease and / or with systemic inflammatory syndrome or sepsis) with a high pro-BNP level.

Starting from all these assumptions we performed this prospective observational study aiming to define the LUS pattern in these categories of acutely ill pediatric patients without primary lung diseases and with high pro-BNP level.

\section{PARTICIPANTS AND METHODS}

\section{Study Population and study setting}

A prospective observational study was conducted in a single tertiary-level teaching hospital. We prospectively analyzed patients aged 1 month to 18 years admitted to the Department of Pediatrics between March 2020 to August 2020.

The enrolled patients were divided into 3 groups: 
Group 1 of the patients under study or group of acutely ill children divided in turn into 3 sub-categories based on the type of disease in progress:

- Cardiac disease

- Systemic inflammatory disease or sepsis without cardiac disease

- Cardiac disease and systemic inflammatory disease/sepsis

Group 2 of children with Bronchiolitis

\section{Group 3 of Healthy Infants}

All patients, belonging to group of acutely ill children and included in the study, underwent anamnestic evaluation and clinical evaluation. Furthermore, laboratory data, airway microbiological data and lung ultrasound data were collected for all. Further investigations were performed only when deemed necessary by the evaluating pediatricians such as chest X-rays and some laboratory tests. During the diagnostic process, the physician on duty made decisions about the patient's diagnosis and treatment according to his/her own practice and without knowledge of the LUS findings, but aware of other clinical / laboratory / imaging data if performed.

Written informed consent was obtained from was obtained by all participants and / or their legal guardians (for children under 16). The study was approved by the Institutional Review Board and Ethic Committee (prot. 36173/19 ID2729). There is no identifying information or pictures in the article.

\section{Patients}

We recruited acutely ill children, aged 1 month to 18 years, and elevated pro-BNP levels (over 300pg/mL) ${ }^{18,19}$ with final diagnosis of A) isolated cardiac disease (with and without heart failure) or B) systemic inflammatory disease / sepsis without heart involvement or C) systemic inflammatory disease and heart involvement as in the case of the acute rheumatic carditis, the Kawasaki disease or the syndrome of pediatric multisystem inflammation temporally related to SARS-CoV-2 (PIMS-TS).

Specifically, at the time of first evaluation, all children with an acute or previous history of heart disease (with and without signs and symptoms of respiratory distress) or with signs and symptoms of suspected systemic inflammatory disease or sepsis (with and without signs and symptoms of respiratory distress) underwent proBNP level assay and lung ultrasound evaluation. Furthermore, all children, belonging to these categories, were subjected to anamnesis, clinical and vital parameters evaluation, evaluation of inflammation indices, always at the first evaluation. In particular, blood tests including C-reactive protein (CRP) and white blood cell count (WCC) and in some cases also procalcitonin (PCT) were performed for all patients. In addition, all were subjected to microbiological tests (molecular and cultural) of the airways to exclude acute upper or lower respiratory tract infections. Further investigations were performed only when deemed necessary by the evaluating pediatricians (troponin dosage, echocardiogram, chest x-ray and other microbiological tests as appropriate).

Finally, keeping in mind the objective of the study - to define whetheracutely ill children with cardiopulmonary interactions had a specific and different lung ultrasound pattern from other categories of patients with primary infectious lung disease and / or completely healthy patients - we enrolled patients belonging to these other two categories. In particular we analyzed the epidemiological and clinical data - as well as the LUS findings - of 1) patients with acute lower respiratory tract infections (in particular bronchiolitisascertained by culture and molecular examinations of the upper airways) aged between 1 month and 2 years and 2) completely healthy infants, then comparing these data with those of the study population of acutely ill children .

Inclusion criteria and stratifications of acutely ill patients

Among the acutely ill patients evaluated, only patients with pro-BNP $>300 \mathrm{pg} / \mathrm{ml}$ and who underwent LUS before the start of any treatment were included. 
Furthermore, acutely ill patients, in addition to being stratified into three sub-categories based on the diagnosis (A) cardiac disease, B) systemic inflammatory disease/sepsis and C) systemic inflammatory disease/sepsis and cardiac disease], were classified into two groups based on the level of pro-BNP, arbitrarily assuming as a cut-off a pro-BNP value of $1000 \mathrm{pg} / \mathrm{ml}$ - level of pro-BNP that several studies have taken as the cut-off value for the clinically significant lung congestion ${ }^{18,19,20,21}$ - to evaluate any changes in epidemiological / clinical / laboratory / instrumental parameters and in LUS findings based on the lowest or highest pro-BNP level of $1000 \mathrm{pg} / \mathrm{ml}$.

Exclusion criteria of acutely ill patients

Instead, we excluded patients belonging to group 1 of our study sample -acutely ill children - but who also had acute upper or lower respiratory tract infections (attested by molecular and cultural microbiological examinations of the airways). In addition, we excluded patients with histories or symptoms and signs of other acute and chronic respiratory diseases (aspiration pneumonia, pneumothorax, pulmonary embolism, interstitial fibrosis, pneumonectomy outcomes, respiratory tract abnormalities), with a history of immunodeficiency, paralysis brain, neuromuscular diseases and malignant tumors because LUS can provide only limited findings to identify a pattern of lung imbibition from non-primarily cardiac causes in these patients.

\section{Lung ultrasound}

LUS was performed with the ultrasound machine ESAOTE MyLab 40, which complies with the Medical Device Directive (MDD) $93 \backslash 42 \backslash$ EEC and subsequent amendments. In accordance with this directive, Esaote has classified it as Class IIa devices. The preset used was small parts. Linear probe $(12-6 \mathrm{MHz})$ was used in preschool children. In older children, we used a curved probe $(8-5 \mathrm{MHz})$. The focus was always positioned at the level of the pleural line. Images and clips were stored and archived. The ultrasound scans were performed by pediatricians and pediatric residents with certifications and / or with an experience of at least 3 years of practice with LUS.

The scans were made by investigating the anterior, lateral and posterior regions of the bilaterally thorax and placing the probe transversally and longitudinally along the lines considered traditional ultrasound findings: the parasternal line, the axillary line and the paravertebral line so as to fully explore the chest wall according to a methodical scheme first described by Copetti et al. ${ }^{22}$

To investigate the anterior and lateral lung fields patients were positioned, according to age, in a seated or supine position. The posterior lung fields have been explored in lateral decubitus and in sitting position.

LUS was performed at the time of first evaluation - and before the start of any treatment - of all children with acute or previous history of heart disease (with and without signs and symptoms of respiratory distress) or with signs and symptoms of suspected disease. systemic inflammatory/sepsis (with and without signs and symptoms of respiratory distress).

All lung regions were scanned with LUS to assess pleural slip and pleural line regularity, presence of A lines, density and characteristics of B lines or vertical artifacts, pleural effusion, and presence of subplerural lung consolidations.

The regular movement of the pleura during breathing defines the pleural sliding which in some circumstances may be absent or have irregularities of the hypercogeneity of the pleruic line. ${ }^{23}$

The A lines were horizontal, regularly spaced, hyperechoic lines representing reverberations of the pleural line (repetition artifacts): this model is considered the physiological lung model. ${ }^{23}$

B lines or vertical artifacts were defined as vertical lines arising from the pleural line. These artifacts show a narrow base, extend to the bottom of the screen without fading, and move synchronously with the lung slide. ${ }^{23}$ However, according to the evidence that the B lines appear different in different pathological and deflationary conditions, depending on the variable distribution of the full-to-void ratio along the pleural line $^{7,24,25}$, in this study the presence of short B lines also have been considered. 
Finally, a fluid-filled anechoic space between the parietal and visceral pleura identified the trasudative pleural effusion $^{1,3,26}$ and lung consolidations were diagnosed by the presence of poorly ventilated or solid images adjacent to the pleura, according to the usual descriptions in the literature. ${ }^{1,3,26}$

The following lung ultrasound finding were detected (Figures 1,2 and 3):

- the presence of short vertical artifacts;

- the presence of long vertical artifacts, their characteristics (multiple or single, confluent or non-confluent, without or with spared areas) and their position (predominantly localized to the pulmonary bases or widespread also in other fields; unilateral or bilateral);

- the presence of anechoic pleural effusion and its location (at the unilateral or bilateral bases);

- the presence of subpleural consolidations;

- the characteristics of the pleural line (regular, irregular, regular with areas of irregularity).

\section{Aims}

Primary aim

To define whether acutely ill children with cardio-pulmonary interactions have a specific and different ultrasound pattern from other categories of patients with primary lung infectious disease or healthy infants (Figures 1,2,3)

Secondary aim

To define LUS patterns of children belonging to the three clinical groups (diagnosis of systemic inflammatory disease / sepsis; diagnosis of cardiac disease; diagnosis of systemic inflammatory disease and cardiac disease) according to the pro-BNP levels (inferior or greater than $1000 \mathrm{pg} / \mathrm{ml}$ )

\section{Statistical analysis}

A Statistical analysis was performed using the software STATA/IC 14.2 version 2017. We provided a description of the set of data using frequency and percentage for categorical variables while continuous variables were described by measures of central tendency (mean or median, depending on whether the distribution was normal or not), and measures of dispersion (standard deviation, SD; interquartile range, IQR). The Shapiro-Wilk test was used to test the normality of the data.

Mainly, we use $\chi 2$ test or Fisher's test for categorical variables as appropriate or Student t test or Mann Whitney test for continuous variables as appropriate.

We considered a 2 -tailed $\mathrm{p}$ value less than 0.05 to be significant.

\section{RESULTS}

\section{Patients' characteristics}

23 acutely ill children with pro-BNP $>300 \mathrm{pg} / \mathrm{mL}$ and suspected pulmonary congestion were enrolled. Of these: -8 diagnosed with systemic inflammatory disease/sepsis (with a median age of 30.5 months); -7 diagnosed with heart disease (with a median age of 147 months); -8 diagnosed with systemic inflammatory disease/sepsis and heart disease (with median age of 127.5 months).

The descriptive analysis of the main epidemiological and clinical characteristics of the study population (acutely ill children), as well as the main diagnostic and laboratory investigations performed on these patients, evaluated according to the three diagnostic sub-category, are described in detail in Table 1.

In addition, the main epidemiological and clinical characteristics of the study population (acutely ill children ), as well as the main diagnostic and laboratory investigations performed on these patients, were assessed according to pro-BNP level group (lower or higher than the value of $1000 \mathrm{pg} / \mathrm{ml}$ ) and are detailed in Table 2. 
The results of the laboratory and other diagnostic investigations were not able to discriminate significantly between the two groups. The only significant difference is that patients who are in the pro-BNP $>1000$ group have a significantly higher frequency of prior cardiac disease $(\mathrm{p}=0.007)$, have significantly higher troponin $(\mathrm{p}=0.002)$, and among the characteristics of echocardiograms have more frequently pathological findings with signs of overload and / or decompensation (92.86\% versus $0 ; \mathrm{p}=0.002)$. Furthermore, $77.78 \%$ of patients belonging to the pro-BNP $<1000$ group have a diagnosis of systemic inflammatory disease/sepsis $(\mathrm{p}=0.003)$.

Finally, we compared the LUS results of the study population (acutely ill children ) with those of a sample of patients suffering from bronchiolitis and a sample of healthy patients. In particular, we analyzed the epidemiological and clinical data - as well as the LUS findings - of 53 patients with bronchiolitis (with a median age of 2 months) and of 33 healthy patients (with a median age of 7 months). The main epidemiological and clinical characteristics of the three populations are detailed in Table 3.

\section{LUS findings}

Details about LUS features in acutely ill children are described in Table 4. Table 4 shows, in particular the differences, of the ultrasound features among acutely ill children, healthy infants and children with bronchiolitis.

Short vertical artifacts were found in $100 \%$ of patients with bronchiolitis and are present in $21.3 \%$ of healthy infants. According to the data published by our study on the analysis of the ultrasound pattern of healthy infants, short vertical artifacts are present during the first 3 months of life in all lung regions and with the passing of the months their presence decreases. ${ }^{2}$ In contrast, in our study population (acutely ill children), no patient presented short vertical artifacts, thus with a significant statistical difference $(\mathrm{p}<0.001)$ compared to bronchiolitis and healthy patients.

Long vertical artifacts are present in $100 \%$ of acutely ill children with a statistically significant difference (p $<0.001)$, particularly compared to healthy patients.

But what is more important, regarding long vertical artifacts, for the qualitative and / or semi quantitative analysis of sonographic interstitial syndrome $e^{7,8,10,25}$ are the differences - in the three groups of patients - of the characteristics and distribution of long vertical artifacts in the different lung fields.

In particular

- $100 \%$ of acutely ill patients have multiple long vertical artifacts with a significant difference $(\mathrm{p}<0.001)$, particularly compared to healthy patients who have single long vertical artifacts in $70.6 \%$ of cases.

- Multiple vertical artifacts are $91.3 \%$ non-confluent inacutely ill children with a statistically significant difference $(\mathrm{p}<0.001)$ compared to the other two groups.

- We found that in acutely ill children, the distribution of long vertical artifacts occurs both "without spared areas" (52.17\% of cases) and "with spread areas" (47.83\%). On the contrary, in $61 \%$ of healthy patients and in $100 \%$ of patients with bronchiolitis, they distribute "with spared areas" in a less homogenous and more densely-packed way. Therefore, also for this ultrasound results, we found statistically significant differences $(\mathrm{p}<0.001)$.

- Another important result concerns the different distributions of long vertical artifacts in the different lung fields explored, which is statistically significant $(\mathrm{p}<0.001)$ in the three groups of patients: in acutely ill children, we found a distribution predominantly in the basal fields $(86.96 \%)$ and also multiple long vertical artifacts are always bilaterally distributed (100\%); on the contrary, in $69.7 \%$ of healthy patients, unilateral distribution prevails with diffusion in all fields explored (87.89\% of cases); as well as in patients with bronchiolitis in $100 \%$ of cases we find long vertical artifacts widespread in all fields explored with a predominantly unilateral $(37.74 \%)$ or bilateral distribution with a prevalent diffusion in the right and / or left lung $(39.62 \%)$. 
We analyzed the presence and distribution of anechoic pleural effusion : it was found only in acutely ill children and in particular in $73.91 \%$ of patients with a bilateral distribution in $41.19 \%$ of cases.

No acutely ill patients enrolled had supleural consolidations or pleural line irregularities. A minority of acutely ill patients $(26.09 \%)$ had a globally regular pleural line with sporadic areas of irregularity. Even in healthy newborns, no subpleural consolidations and irregularities of the pleura line were found in any patient, on the contrary in patients with bronchiolitis subpleural consolidations and irregularities of the pleural line were found in $100 \%$ of patients.

Table 5 shows the differences in ultrasound results in the two groups of acutely ill children with pro-BNP level lower and higher than $1000 \mathrm{pg} / \mathrm{ml}$. For many of the ultrasound findings, there are no statistically significant differences in the two groups. We found a significant difference $(\mathrm{p}<0.001)$ in the distribution of long vertical artifacts "with or without spared areas". In particular, we reconsidered that $100 \%$ of acutely ill patients with pro-BNP $<1000 \mathrm{pg} / \mathrm{ml}$ present a destruction of long vertical artifacts "with spared areas". Most children with pro-BNP $<1000$ belong to the group of acutely ill children with systemic inflammatory disease/sepsis in the absence of organic and / or functional damages in the myocardium (Table 1 and Table 2 ). In $85.71 \%$ of cases of the children belonging to the group with pro-BNP $>1000 \mathrm{pg} / \mathrm{ml}$ (most of these belong to the heart disease group with or without systemic inflammation/sepsis as shown in Table 1 and Table 2) vertical artifacts are distributed homogeneously "without spared areas " (as from early cardiogenic edema).

Table 6 shows the differences of the ultrasound features in the three diagnostic categories of acutely ill children .

\section{DISCUSSION}

Our prospective observational study is the first study that evaluates the LUS findings of cardiopulmonary interactions in acutely ill children with elevated pro-BNP levels, with the aim of establishing the specific LUS pattern in this category of patients without primary lung diseases.

We found that LUS findings in these acutely ill children are different from the ultrasound pattern of other categories of children and in particular 1) children with acute lower respiratory tract infections and 2) healthy infants (Figures 1,2 and 3). In particular, we found that no children belonging to the category ofacutely ill children had short vertical artifacts but all had multiple long artifacts that were well delineated and non-blurred, non-confluent, with localization mainly at the bases and bilateral distribution. At the origin of these artifacts, the pleural line always appeared regular and none had subpleural consolidations. Many had anechoic pleural effusions, most of them unilateral.

We also found that the distribution pattern of vertical artifacts (with or without spared areas) is influenced not only by the cause, severity and timing of onset of pulmonary congestion - as already described in the literature ${ }^{7,8,10}$ - but also by the level of pro-BNP and the type of pathology (cardiac disease; systemic inflammatory disease/sepsis; heart disease and systemic inflammatory disease/sepsis) that causes the increase in pro-BNP (Tables 5 and 6). However, due to the scarcity of the comparison population, we can consider this last detection as a preliminary result pending the execution of a study with a larger sample of patients, in order to understand if there is a correlation between the ultrasound results and their distribution with pro-BNP levels and / or specific diagnosis.

From a theoretical point of view, our results reinforce the need for a better understanding of the physical mechanisms that generate $\operatorname{artifacts}^{27}$ even in children.

In the presence of cardiogenic edema, ARDS, interstitial lung diseases, bronchiolitis and non-consolidating pneumonia and contusions, part of the lung volume originally occupied by air, can be replaced with water, connective, cells, hyaline membrane or edematous tissue, eventually creating acoustic traps for the US beam containing a medium that is physically (in terms of acoustic impedance) very different from the surrounding environment (air). ${ }^{7,8,10,25}$ 
Therefore, the B lines, in their variable appearances, indicated a loss of peripheral lung aeration (without tissue consolidation) due to interstitial disease or simply to lung deflation without histologic changes. ${ }^{7,8,10,25}$ However, the B lines, in the absence of an analysis of their appearance, cannot easily differentiate the cause. ${ }^{7,8,10,12,25}$ Studies performed on physical models and in adult patients ${ }^{7-12,21,25}$, have shown that the vertical artifacts generated by an inflammatory lung have a different appearance from those generated by cardiogenic edema and / or pulmonary congestion not primarily due to lung diseases. ${ }^{7-12,21,25}$

In the first case, as for example in the context of lung inflammation or in ARDS, the random peri and intralobular distortion can explain the appearance of acoustically permissive irregular channels and consequently the appearance of different arrangements of the B lines, pleural irregularities, consolidations, inhomogeneous isolated air spaces and inhomogeneous edema. ${ }^{7,8,10,25}$ Inhomogeneous edema, alveolar collapses, fibrosis and lung inflammation contribute to the development of pleural irregularities and the reduction of lung compliance and pleural flow in ARDS and other lung inflammatory diseases.

In the second case, the presence of homogeneously distributed edema due to the absence of inflammation and / or fibrosis causes the secondary interlobular septa to be thickened but anatomically intact and therefore can act as acoustic traps in which specific frequencies give rise to separate B lines, uniform, bright and long, associated with regular pleural line, with no spared areas especially in early cardiogenic edema. ${ }^{7,8,10,25}$

When cardiogenic pulmonary edema is severe or in an advanced stage, some LUS findings can be superimposed on the LUS findings of ARDS such as: - irregularities of the pleural line with small supleural consolidations or - the presence of "spared areas". 8,9

None of the acutely ill patients that we enrolled for the study had a clinical and functional picture of ARDS: in fact, these are patients with isolated heart disease with early cardiogenic pulmonary edema and patients with systemic inflammatory syndrome and / or sepsis who did not have such a severe picture as to develop ARDS. In agreement with the data in the literature ${ }^{8,9}$, in none of the acutely ill patients we found short vertical artifacts, pleuric line irregularities and sub pleural consolidations.

Furthermore, a recently published ${ }^{2}$ study showed that healthy young children can also generate vertical artifacts and this discovery has provided us with new information and a new group of patients to study the $\mathrm{B}$ lines. We have in fact applied these new results in the design. and in the execution of our study.

From a practical point of view, our study confirms what we have learned so far from these preclinical and clinical studies. ${ }^{7-12,21,25}$ It underlines the idea that vertical artifacts must be interpreted according to the clinical scenario case by case and how through this interpretation it is possible to find specific patterns for some categories of patients different from each other in order to use LUS as an aid to orient ourselves more easily in the differential diagnosis of the pathologies that are accumulated by the presence of SIS.

In particular, in consideration of the finding of statistically significant differences (Table 4) for all the ultrasound findings studied between the group of acutely ill children compared to that of bronchiolitis but also compared to that of healthy patients, it was possible to find a specific ultrasound pattern of cardiopulmonary interactions.

This means that the finding in a child of a SIS with multiple, bright, long, separate and non-confluent B-lines/long vertical artifacts deriving from a normal and regular pleural line, in the absence of subpleural consolidations, is strongly predictive of cardiogenic pulmonary edema or pulmonary congestion in the course of systemic inflammatory disease/sepsis (Figure 1).

Regarding the latter subcategory of acutely ill of patients, in our clinical practice we have observed elevated levels of pro-BNP and lung ultrasound findings suggesting a pulmonary imbibition picture even in patients with systemic inflammatory pathology / sepsis in the absence of cardiac dysfunction. Therefore we have decided to include in the category of acutely ill children not only patients with heart disease but also those with systemic inflammatory pathology with and without cardiac dysfunction (such as rheumatic carditis, Kawasaki disease, PIMS-ST, sepsis) with high levels of pro-BNP. 
BNP and NT-proBNP are released into the bloodstream by cardiomyocytes in response to stress and pressure placed on the atria and ventricles. pro-BNP is a biomarker of cardiac overload associated with the severity of cardiovascular disease ${ }^{14}$ and the severity of sepsis. ${ }^{15,16,17}$ It has long been proven that patients show signs of heart failure during sepsis. ${ }^{14-17}$ Indeed, the destructive systemic inflammatory response and intensive resuscitation occurring during sepsis result in widespread organ overload and damage, including myocardial damage. ${ }^{15-17}$

However, today we know that in the course of sepsis and / or systemic inflammatory disease, the increase in plasma levels of pro-BNP can be present even in the absence of damage or dysfunction on the cardiomyocytes. ${ }^{16,17}$ In particular, Meader and colleagues and by Renana and colleagues ${ }^{16,28}$, have shown that in patients with sepsis, the level of BNP in the blood increases even without heart failure and that it is positively correlated with the level of CRP, suggesting the role of inflammation in the increase of BNP. ${ }^{16,28}$ Furthermore, experimental studies ${ }^{17,29}$ have shown that endotoxins and pro-inflammatory cytokines released in conspicuous quantities during sepsis or systemic inflammation, as well as playing a direct role on cardiac toxicity, directly increase the gene expression of the BNP in cardiomyocytes. This would explain the higher levels of this biomarker (positively correlated with the level of CRP and IL-6), in systemic inflammatory processes even in the absence of significant organic or functional cardiac alterations. ${ }^{16,17,28,29}$

In this sub-category of acutely ill of patients with systemic inflammatory disease / sepsis, we found a lung ultrasound picture of pulmonary congestion. But, although none of our enrolled acutely ill children developed ARDS during sepsis and / or systemic inflammatory disease, we know that these patients can also develop an inflammatory of ARDS pulmonary picture ${ }^{9,10,30}$ especially in the most severe cases. In these cases, according to what emerged from our study, LUS would support the clinic and laboratory data in distinguishing the two conditions with different ultrasound patterns - congestion and inflammation - with clinical implications, important diagnostics and therapeutics.

We have also found that the distribution pattern of long vertical artifacts ("with or without spared areas") is influenced not only by the severity and time of onset of pulmonary congestion ${ }^{8}$, but also by the level of pro-BNP and the type of pathology (cardiac disease, systemic inflammatory disease / sepsis, cardiac disease and systemic inflammatory disease / sepsis) which causes the increase in pro-BNP (Tables 5 and 6).

In fact, in the literature there is discussion on homogeneous septal distribution "without spared areas" in the case of early cardiogenic edema. In the later stages of pulmonary edema, the thickening of the interlobular septa becomes excessive and goes beyond their capacity, therefore alveolar flooding occurs when the secondary interlobular septae are maximally distended by the transudative fluid. Flooding and alveolar instability, together with the collapse of peripheral airspace, generate many different types of acustic channels and consequently different artifacts with many arrangements. Therefore, pulmonary cardiogenic edema, in the later stages of the pathology, can lose the ultrasound characteristic of the septal and homogeneous B lines and increasingly tend to resemble the edema of early ARDS without consolidation "with spared areas". ${ }^{8,9,10}$

We found that in $85.71 \%$ of cases of children belonging to the group with proBNP> $1000 \mathrm{pg} / \mathrm{ml}$ (most of these belong to the group of heart disease with or without systemic inflammation / sepsis as shown in Table 1 and Table 2 ) vertical artifacts are homogeneously distributed "without spared areas" (as from early cardiogenic edema). On the other hand, $100 \%$ of acutely ill patients with pro-BNP $<1000 \mathrm{pg} / \mathrm{ml}$ have destruction of long vertical artifacts "with spared areas" (Table 5). However, the majority of children with pro-BNP $<1000$ belong to the group of acutely ill children with systemic inflammatory disease / sepsis in the absence of organic and / or functional damage in the myocardium (Table 1, Table 3). Table 6 also shows that $87.5 \%$ of patients with inflammatory pathology and / or sepsis in the absence of cardiac dysfunction have a distribution of artifacts "with spared areas". We therefore hypothesized that in this subcategory of patients the distribution of artifacts can also be influenced by the underlying inflammatory process. Due to the scarcity of the comparison population, we can consider these results as preliminary pending the execution of a study on a larger sample of patients, in order to understand if there is a correlation between the ultrasound results, their distribution and BNP levels and the type of cardiac and / or inflammatory disease (Tables 5 and 6). 
Recent studies and systematic reviews performed in adult patients have shown that LUS can identify acute heart failure and pulmonary edema with high sensitivity and specificity, demonstrating greater sensitivity and specificity than chest x-ray. ${ }^{11,12,13}$

With our study we could not compare the ultrasound results with the X-ray results because not all acutely ill children performed chest X-rays. It has not been performed for example in children with a diagnosis of heart disease already known or for those children for whom it was not necessary to perform it by virtue of the diagnostic suspicion already clear from the initial symptoms as in some cases of systemic inflammatory disease/sepsis. Others, however, in particular children with a diagnosis of not known heart disease in the acute phase (such as acute heart failure in the course of unknown heart disease or acute rheumatic carditis not known with atrioventricular overload) who presented in the emergency room with cough and dyspnea or those presenting with persistent fever (with subsequent diagnosis of systemic inflammatory disease/sepsis) underwent chest $\mathrm{x}$-rays to rule out infectious respiratory disease.

As has happened in our clinical practice, -in some cases (as in the case of mild and early pulmonary edema), the x-ray can be completely negative; - in other cases (such as in the presence of pleural effusion with consensual atelectasis), chest x-ray may be a confounding factor in suspecting an quadro of pleuropneumonia; - and in still other cases (in children who present a febrile clinical picture in the context of a systemic inflammatory disease / sepsis) chest X-ray can once again erroneously suspect a suspected viral infection and / or bacterial of the lower airways as responsible for fever peaks. ${ }^{3,4,12}$

All this obviously has implications in clinical practice: using only the chest X-ray in the initial phase of the diagnostic process, there is a risk of making an incorrect diagnosis with the risk of delaying the correct diagnosis on one side and on the other side of administering unnecessary therapies by making inappropriate use especially of antibiotic therapy.

Therefore, in addition to the now well-known advantages of LUS in the pediatric population (non-invasive, non-ionizing radiation tool characterized by a rapid, affordable, point-of-care imaging modality that allow both real-time diagnosis and follow-up of respiratory diseases ${ }^{31-34}$ ), integrating LUS in the earliest phase of the diagnostic process of acutely ill patients also in the pediatric population allows for a greater possibility of earlier diagnosis and appropriate diagnosis in light of the result of the specific lung ultrasound pattern such as our results prove.

Our study has some limitations. First of all, due to the COVID-19 pandemic we have been able to enroll and evaluate a small number of acutely ill patients.

Secondly, having a small patient population, despite being a heterogeneous patient population by age, we were unable to stratify patients according to age and evaluate differences with respect to LUS findings and their distribution.

Finally, due to the scarcity of the comparison population, we could not adequately analyze the presence or absence of correlations between the ultrasound parameters analyzed, their distribution and the type of pathology (cardiac disease, systemic inflammatory disease, cardiac diasease and systemic inflammatory disease) and the values of pro-BNP, thus obtaining preliminary results regarding this aspect.

In conclusions, the LUS pattern we described in our "acutely ill children" population is in line with recent advances in SIS analysis in preclinical models and adult patients with respiratory disease and/or with cardiopulmonary interactions and reinforces the need to focus more efforts on studies of LUS artifacts also on the pediatric population.

Dedicated software, algorithms and transducers, and artificial intelligence are all needed to explore the information contained in US signals. ${ }^{35}$ However, pending the development and testing of new software that can obtain objective information (quantitative and qualitative), our results confirm that the knowledge of the different origin and the histological characteristics of the B lines allows us to perform a qualitative and semi-quantitative analysis of the SIS. In fact now, point-of-care physicians and sonographers can also apply our results in the first-line work-up in the diagnostic approach to this category of patients. 
To date, it remains fundamental to have the clinical and laboratory background of each patient as a guideline in the interpretation of SIS even in acutely ill children. Indeed, new studies, including preclinical ones along with the use of artificial intelligence, are needed to validate our results.

\section{CONFLICT OF INTERESTS}

The authors declare that there are no conflict of interests.

\section{FUNDING}

The authors declare that there are no funding.

\section{AUTHORS CONTRIBUTION}

DB, CDR and PV conceptualized the study. DB, CDR, RM, and AM were responsible for data

collection, and patient care. VF performed the statistical analyses. All authors contributed to the draft of the manuscript and agreed with the final version of the manuscript.

\section{REFERENCES}

1. Tomà P. Lung ultrasound in pediatric radiology - cons. Pediatr Radiol . 2020;50(3):314-320.

2. Buonsenso D, Soldati G, Curatola A, et al. Lung Ultrasound Pattern in Healthy Infants During the First 6 Months of Life. J Ultrasound Med . 2020;39(12):2379-2388.

3. Berce V, Tomazin M, Gorenjak M, Berce T, Lovrenčič B. The Usefulness of Lung Ultrasound for the Aetiological Diagnosis of Community-Acquired Pneumonia in Children. Sci Rep . 2019;9(1):17957.

4. Buonsenso D, Musolino A, Ferro V, et al. Role of lung ultrasound for the etiological diagnosis of acute lower respiratory tract infection (ALRTI) in children: a prospective study. $J$ Ultrasound . 2021;10.1007/s40477-021-00600-z.

5. Liu J, Lovrenski J, Ye Hlaing A, Kurepa D. Neonatal lung diseases: lung ultrasound or chest x-ray. $J$ Matern Fetal Neonatal Med . 2021;34(7):1177-1182.

6. Supino MC, Buonsenso D, Scateni S, et al. Point-of-care lung ultrasound in infants with bronchiolitis in the pediatric emergency department: a prospective study. Eur J Pediatr . 2019;178(5):623-632.

7. Soldati G, Demi M, Inchingolo R, Smargiassi A, Demi L. On the Physical Basis of Pulmonary Sonographic Interstitial Syndrome. J Ultrasound Med . 2016;35(10):2075-2086.

8. Soldati G, Demi M, Demi L. Ultrasound patterns of pulmonary edema. Ann Transl Med . 2019;7(Suppl 1):S16.

9. Copetti R, Soldati G, Copetti P. Chest sonography: a useful tool to differentiate acute cardiogenic pulmonary edema from acute respiratory distress syndrome. Cardiovasc Ultrasound . 2008;6:16.

10. Soldati G, Demi M. The use of lung ultrasound images for the differential diagnosis of pulmonary and cardiac interstitial pathology. J Ultrasound . 2017;20(2):91-96.

11. Nakao S, Vaillancourt C, Taljaard M, Nemnom MJ, Woo MY, Stiell IG. Diagnostic Accuracy of Lung Point-Of-Care Ultrasonography for Acute Heart Failure Compared With Chest X-Ray Study Among Dyspneic Older Patients in the Emergency Department. J Emerg Med . 2021;S0736-4679(21)00154-2.

12. Maw AM, Hassanin A, Ho PM, et al. Diagnostic Accuracy of Point-of-Care Lung Ultrasonography and Chest Radiography in Adults With Symptoms Suggestive of Acute Decompensated Heart Failure: A Systematic Review and Meta-analysis. JAMA Netw Open . 2019;2(3):e190703.

13. Sartini S, Frizzi J, Borselli M, et al. Which method is best for an early accurate diagnosis of acute heart failure? Comparison between lung ultrasound, chest X-ray and NT pro-BNP performance: a prospective study. Intern Emerg Med . 2017;12(6):861-869.

14. Kirchhoff C, Leidel BA, Kirchhoff S, et al. Analysis of N-terminal pro-B-type natriuretic peptide and cardiac index in multiple injured patients: a prospective cohort study. Crit Care . 2008;12(5):R118

15. Tsai SH, Lin YY, Chu SJ, Hsu CW, Cheng SM. Interpretation and use of natriuretic peptides in non-congestive heart failure settings. Yonsei Med J . 2010;51(2):151-163.

16. Li N, Zhang Y, Fan S, Xing J, Liu H. BNP and NT-proBNP levels in patients with sepsis. Front Biosci (Landmark Ed) . 2013;18:1237-1243. 
17. Custodero C, Wu Q, Ghita GL, et al. Prognostic value of NT-proBNP levels in the acute phase of sepsis on lower long-term physical function and muscle strength in sepsis survivors. Crit Care . 2019;23(1):230.

18. Swedberg K, Cleland J, Dargie H, et al. Guidelines for the diagnosis and treatment of chronic heart failure: executive summary (update 2005): The Task Force for the Diagnosis and Treatment of Chronic Heart Failure of the European Society of Cardiology. Eur Heart J . 2005;26(11):1115-1140.

19. Januzzi JL, van Kimmenade R, Lainchbury J, et al. NT-proBNP testing for diagnosis and short-term prognosis in acute destabilized heart failure: an international pooled analysis of 1256 patients: the International Collaborative of NT-proBNP Study. Eur Heart J . 2006;27(3):330-337.

20. Tsai SH, Lin YY, Chu SJ, Hsu CW, Cheng SM. Interpretation and use of natriuretic peptides in non-congestive heart failure settings. Yonsei Med $J$. 2010;51(2):151-163.

21. Miglioranza MH, Picano E, Badano LP, et al. Pulmonary congestion evaluated by lung ultrasound predicts decompensation in heart failure outpatients. Int J Cardiol . 2017;240:271-278.

22. Copetti R, Cattarossi L. Ultrasound diagnosis of pneumonia in children. Radiol Med . 2008;113(2):190198.

23. Volpicelli G, Elbarbary M, Blaivas M, et al. International evidence-based recommendations for pointof-care lung ultrasound. Intensive Care Med . 2012;38(4):577-591.

24. Demi M, Prediletto R, Soldati G, Demi L. Physical Mechanisms Providing Clinical Information From Ultrasound Lung Images: Hypotheses and Early Confirmations. IEEE Trans Ultrason Ferroelectr Freq Control . 2020;67(3):612-623.

25. Soldati G, Demi M, Smargiassi A, Inchingolo R, Demi L. The role of ultrasound lung artifacts in the diagnosis of respiratory diseases. Expert Rev Respir Med. 2019 Feb;13(2):163-172. doi: 10.1080/17476348.2019.1565997.

26. Lichtenstein DA. Ultrasound in the management of thoracic disease. Crit Care Med . 2007;35(5 Suppl):S250-S261.

27. Demi L, van Hoeve W, van Sloun RJG, Soldati G, Demi M. Determination of a potential quantitative measure of the state of the lung using lung ultrasound spectroscopy. Sci Rep . 2017;7(1):12746.

28. Shor R, Rozenman Y, Bolshinsky A, et al. BNP in septic patients without systolic myocardial dysfunction. Eur J Intern Med . 2006;17(8):536-540.

29. de Bold AJ. Cardiac natriuretic peptides gene expression and secretion in inflammation. J Investig Med. 2009;57(1):29-32.

30. Zhang J, Luo Y, Wang X, et al. Global transcriptional regulation of STAT3- and MYC-mediated sepsis-induced ARDS. Ther Adv Respir Dis . 2019;13:1753466619879840.

31. Volpicelli G. Lung sonography. J Ultrasound Med . 2013;32(1):165-171.

32. Musolino AM, Tomà $\mathrm{P}$, Supino $\mathrm{MC}$, et al. Lung ultrasound features of children with complicated and noncomplicated community acquired pneumonia: A prospective study. Pediatr Pulmonol . 2019;54(9):1479-1486.

33. Buonsenso D, Brancato F, Valentini P, Curatola A, Supino M, Musolino AM. The Use of Lung Ultrasound to Monitor the Antibiotic Response of Community-Acquired Pneumonia in Children: A Preliminary Hypothesis. J Ultrasound Med . 2020;39(4):817-826.

34. Cattarossi L, Copetti R, Poskurica B. Radiation exposure early in life can be reduced by lung ultrasound. Chest . 2011;139(3):730-731.

35. Brusasco C, Santori G, Bruzzo E, et al. Quantitative lung ultrasonography: a putative new algorithm for automatic detection and quantification of B-lines. Crit Care . 2019;23(1):288.

\section{FIGURE LEGENDS}

FIGURE 1. Grayscale lung ultrasound examination (linear probe, $12 \mathrm{MHz}$, and the small parts preset) shows Septal Pattern of early CPE (8) in a 14-year-old boy with acute heart failure caused by non-diagnostic and untreated rheumatic carditis (A and B) and in a 5-year-old girl with systemic inflammatory disease in course of sepsis in the absence of functional and / or organic alteration of the myocardium (C, D and E).

Figures A and B show the SIS pattern of the posterior basal right and left fields respectively; those C, D and 
E show that of the posterior basal left and right fields (C and D) and lateral basal right (E) respectively.

The pleural line is regular (arrowhead). There are no short vertical artifacts and subpleural consolidations. B-lines or long vertical artifacts (arrows) are multiple, and separated / non-confluent, laser like artefacts, with gravitational course spreading from the pleural line to the bottom of the screen and they show an internal sequence of alternating horizontal bands. They are distributed homogeneously in the explored fields "without spared areas".

CPE, cardiogenic pulmonary edema; SIS, sonographic interstitial syndrome.

FIGURE 2. Grayscale lung ultrasound examination (linear probe, $12 \mathrm{MHz}$, and the small parts preset) in a child of 14 months with viral lower respiratory tract infection caused by RSV and Bocavirus. The figures above and below show the SIS pattern located on the upper and lower fields respectively of the left posterior lung fields.

The pleural line is irregular. There are small subpleural consolidations (hypoechoic areas, arrows) and below areas of white are evident (arrowheads). B-lines or long vertical artifacts are multiple and confluent; they do not have a gravitational trend and do not have a laser arefacts-like appearance; they create areas of white lung (arrowheads) and are unevenly distributed in the explored fields.

RSV, Respiratory Syncytial Virus; SIS, sonographic interstitial syndrome.

FIGURE 3. Grayscale lung ultrasound examination (linear probe, $12 \mathrm{MHz}$, and the small parts preset) shows a SIS picture in a completely healthy infant.

Figures A and B show the SIS pattern of the left anterior upper and lower fields respectively; those C, D show that of the upper posterior left and upper lateral left fields respectively.

The pleural line is regular (arrowheads) with no subpleural consolidations. Figures B and D show short vertical artifacts (black arrows). B-lines or long vertical artifacts (withe arrows) are single and separated / non-confluent in figures $\mathrm{A}$ and $\mathrm{B}$, while in figures $\mathrm{C}$ and $\mathrm{D}$ they are multiple and tend to merge. They are distributed unevenly "with spared areas" where a normal ultrasound pattern with A lines (asteristisks) is clearly visible.

SIS, sonographic interstitial syndrome. 


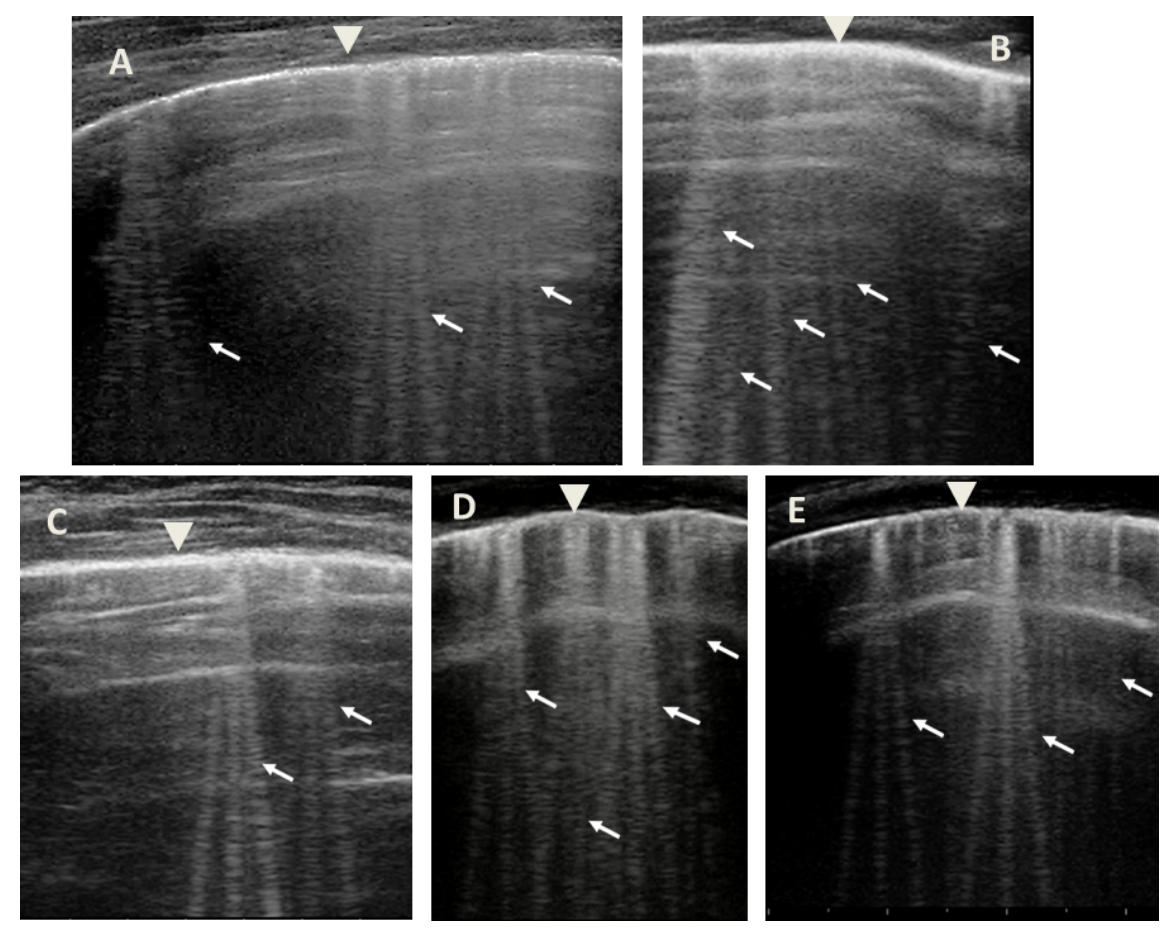




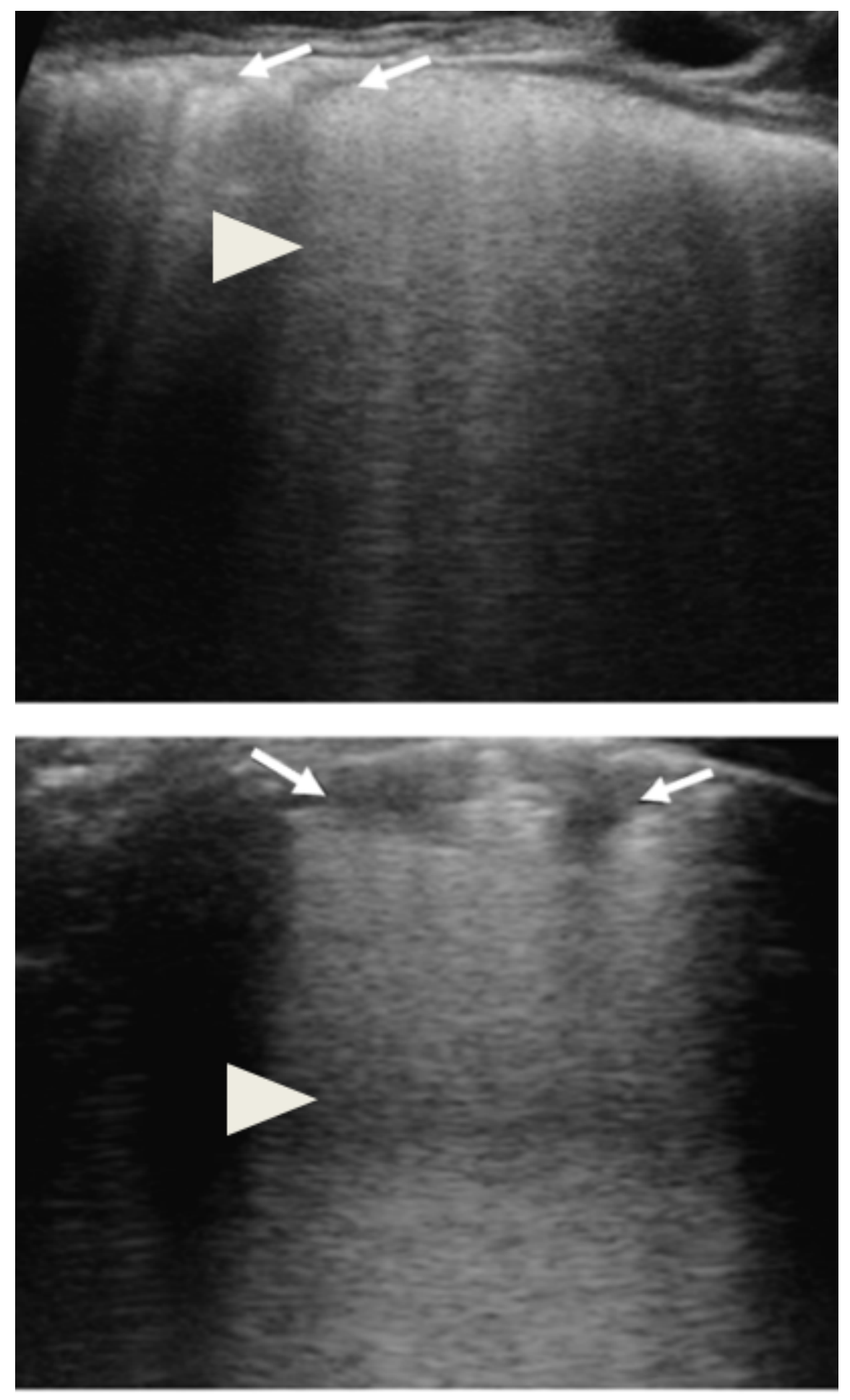



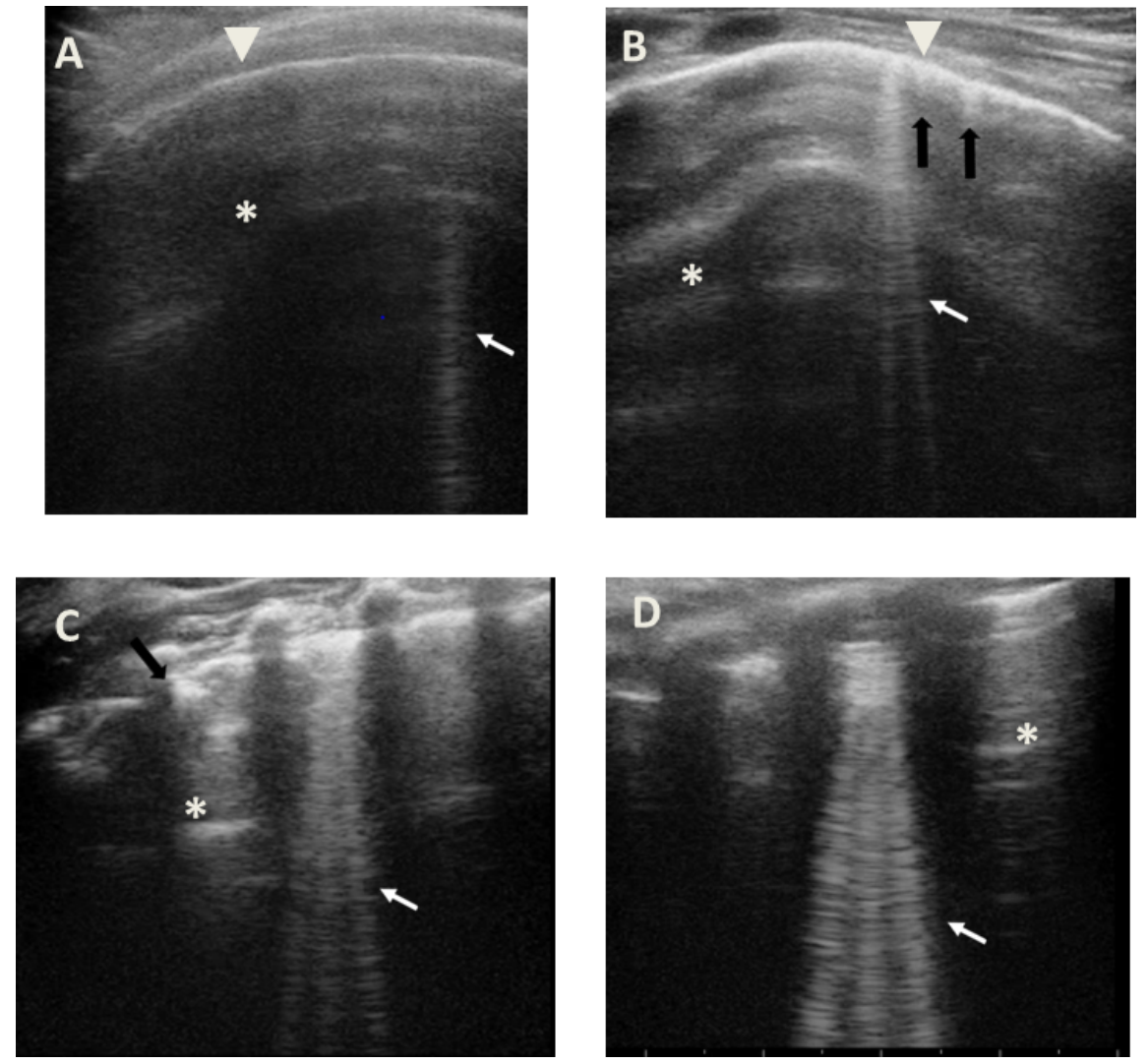

\section{Hosted file}

Tables.docx available at https://authorea.com/users/363303/articles/528381-lung-ultrasoundto-detect-cardiopulmonary-interactions-in-acutely-ill-children 